\title{
Quantum Chemical Study on the Corrosion Inhibition of Copper Using Some Thiosemicarbazides and Tetrazoles
}

\begin{abstract}
Relationships between corrosion inhibition efficiency of two thiosemicarbazide based compounds namely; 4phenyl thiosemicarbazide (PTSC), Salicylaldehyde thiosemicarbazone (STSC) and 4 tetrazole derivative; 5Mercapto-1-methyltetrazole (MMTA), 5-(3-Pyridyl)-1H-tetrazole (PyTA), 5-Amino-tetrazole mono hydrate (ATA) and 5-phenyl tetrazole (PTA) and their molecular electronic properties have been theoretically studied at the level of DFT/B3LYP with $6-31+\mathrm{G}(2 \mathrm{~d}, 2 \mathrm{p})$ base sets. The structural parameters, such as energy, highest occupied molecular orbital (HOMO) energy and lowest unoccupied molecular orbital (LUMO) energy, the charge distribution of the studied inhibitors, the absolute electronegativity $(\chi)$ values were calculated and discussed using linear regression.
\end{abstract}

Abdul-Khalik Younis ${ }^{1}$, Ibrahim M. Ghayad ${ }^{2}$, ${ }^{*}$, Fatma Kandemirli ${ }^{1}$,

${ }^{1}$ Department of Materials Science \& Engineering, Fac. of Engineering and Architecture, Kastamonu University, 3720, Turkey

${ }^{2}$ Central Metallurgical Research \& Development Institute, P.O. Box: 87, Helwan, Cairo, Egypt

*Corresponding author e-mail: ighayad@yahoo.com

Keywords: Thiosemicarbazides and Tetrazoles, structural parameters

DOI: $10.7176 / \mathrm{CMR} / 11-2-04$

\section{Introduction}

Organic compounds having a $\pi$ bond and a heteroatom such Oxygen; Nitrogen and Sulfur have been widely used [1-3] as corrosion inhibitors. In general, the inhibitor molecule may be physically or chemically adsorbed on the corrosive metal surface. The adsorption is usually formed on the metal surface where the adsorbent layer serves as a barrier protecting metals from corrosion $[4,5]$.

It is widely recognized that organic inhibitors typically promote the formation of chelates on the metal surface, by transferring electrons from the organic compound to the metal and forming a coordination covalent bond during the chemical adsorption process $[6,7]$. Factors that influence the adsorption of these molecules, such as functional groups, space factors, molecular size, electron density on donor atoms (such as, N, S, P, and O), and orbital characteristics of donor electrons, have been considered and discussed. The inhibitory efficiency is closely related to the ability of the inhibitor to adsorb and the molecular properties of the different species of organic compounds [8-10]. Heterocyclic compounds, kind of effective inhibitors [11-14], have at least two factors within their structures, which is the reason of their effective inhibiting. The planarity $(\Pi)$ and the lonely electron pairs in the heteroatoms are important features that determine the adsorption of these molecules on the metallic surface by transferring electrons from the organic compounds to the metal and forming a coordinate covalent bond during the chemical adsorption. Recently, tetrazoles derivatives have been used as preservatives for copper alloys [15-17].

Recently, the use of theoretical chemistry to explain the mechanism of corrosion inhibition, such as quantum chemistry calculations, has proved to be a very powerful tool for studying the mechanism [18-19]. Previous studies have been shown that the corrosion inhibition efficiency with molecular properties for different kinds of organic compounds is very important factor in quantum mechanical studies [20-22].

There are many applications in using the HOMO-LUMO gap as a quantum descriptor for establishing correlations in various chemical and biochemical systems. Quantum chemical descriptors such as chemical potential, chemical hardness, softness, electrophilic index and Fukii function have been widely used to explain the mechanism of corrosion inhibition [23-29].

The purpose of this study is to propose theoretical studies on the electronic and molecular structures for some thiosemicarbazide and tetrazole derivatives and determine the relationship between the quantum chemical descriptor parameter obtained from the molecular structure of the compound and the inhibition efficiency of the compounds. 


\section{Theory and Computational Details}

Density functional theory (DFT) methods were used in this study. These methods have become very popular in recent years because they can reach exactitude similar to other methods in less time and they are less expensive from the computational point of view. In agreement with the DFT results, energy of the fundamental state of a polyelectronic system can be expressed through the total electronic density, and, in fact, the use of electronic density instead of wave function for calculating the energy constitutes the fundamental base of DFT [30]. All the calculations were done by GAUSSIAN $03 \mathrm{~W}$ software [31], using the B3LYP functional [31,32] and a 6-31G* basis set [33]. The B3LYP, a version of DFT method, uses Beche's three-parameter functional (B3) and includes a mixture of HF with DFT exchange terms associated with the gradient corrected correlation functional of Lee, Yang, and Parr (LYP) [31,32]. This approach is shown to yield favorable geometries for a wide variety of systems.

\section{Results and Discussion}

Tables 1\&2 and Figures 1-3 show the calculated parameters of the inhibitors molecules using B3LYP/6-311G(d,p), B3LYP/6-311++G(2d,2p) methods. The frontier orbital energy of PyTA calculated by B3LYP / 6-311G $(d, p)$ in

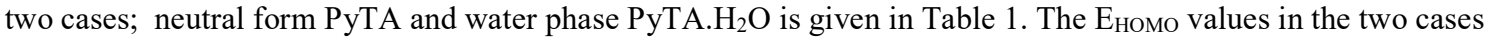
are: -7.633 and $-7.369 \mathrm{eV}$, respectively. The $\mathrm{E}_{\mathrm{Lumo}}$ values in the two cases are: -2.33 and -1.926 , respectively.

The frontier orbital energy of ATA calculated by B3LYP / 6-311G $(d, p)$ in two cases; ATA, ATA.H $\mathrm{H}_{2} \mathrm{O}$ is given

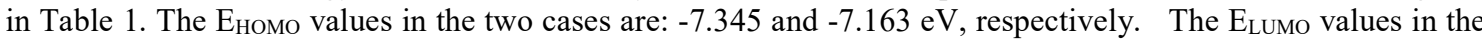
two cases are: -1.003 and -.702 , respectively.

The frontier orbital energy of MMTA calculated by B3LYP / 6-311G (d, p) in two cases; 2-PyTA, PyTA-w is

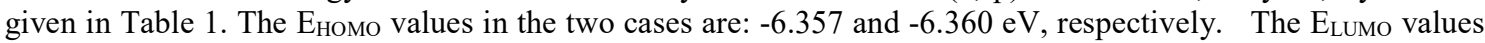
in the two cases are: -2.492 and -2.091 , respectively.

The frontier orbital energy of PTSC calculated by B3LYP / 6-311G (d, p) in two cases ; PTSC, PTSC- $\mathrm{H}_{2} \mathrm{O}$ is given

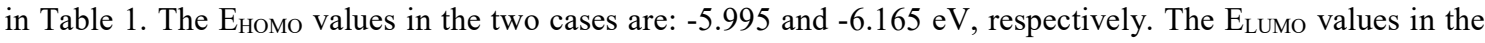
two cases are: --1.052 and -1.054, respectively.

The frontier orbital energy of PTA calculated by B3LYP / 6-311G (d, p) in two cases ; PTA, PTA.H $\mathrm{H}_{2}$ is given in Table 1. The Еномо values in the two cases are: -6.794 and $-6.913 \mathrm{eV}$, respectively. The ELumo values in the two cases are: -1.351 and -1.430 , respectively.

The frontier orbital energy of STSC calculated by B3LYP / 6-311G (d, p) in two cases ; STSC, STSC- $\mathrm{H}_{2} \mathrm{O}$ is

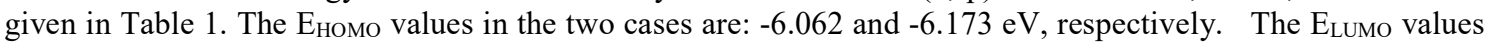
in the two cases are: -1.099 and -1.076 , respectively.

As HOMO is often associated with the electron donating ability of a molecule, high value of HOMO is likely to indicate the tendency of the molecule to donate electrons to appropriate acceptor molecules with lower energy MO. The calculated HOMO energies of the six molecules are: $-5.995 \mathrm{eV},-6.794,-6.062,-7.633,-7.345,-6.357 \mathrm{eV}$ for PTSC, PTA and STSC, PyTA, ATA and MMTA, respectively, while the LUMO energies are: $-1.052 \mathrm{eV}$, $1.351,-1.099,-2.233,-1.003,-2.492 \mathrm{eV}$ for PTSC, PTA and STSC, PyTA, ATA and MMTA, respectively. On the other hand, the energy gaps were $4.942,5.443,4.963,5.400,6.342,3.865 \mathrm{eV}$, respectively.

It is postulated that the inhibition efficiency is increased with the increase in HOMO, the decrease in LUMO and the increase in the energy gap. Accordingly, the descending order of inhibition efficiency will be as follows: ATA $>$ PTA $>$ PyTA $>$ STSC $>$ PTSC $>$ MMTA.

According to the frontier molecular orbital theory (FMO) of chemical reactivity, transition of electron is due to interaction between highest occupied molecular orbital (HOMO) and lowest unoccupied molecular orbital (LUMO) of reacting species [30]. The inhibitor does not only donate electron to the unoccupied d orbital of the metal ion but can also accept electron from the d-orbital of the metal leading to the formation of a feedback bond. The energy of the lowest unoccupied molecular orbital, ELUMO, indicates the ability of the molecule to accept electrons. The binding ability of the inhibitor to the metal surface increases with increasing of the HOMO and decreasing of the LUMO energy values. Therefore, the lower the value of ELUMO is the more probable the molecule to accept electrons. Energy gap is an important parameter as a function of reactivity of the inhibitor molecule toward the adsorption on the metallic surface. As $\Delta \mathrm{E}$ decreases, the reactivity of the molecule increases leading to increase in the \% IE of the molecule. Lower values of the energy difference will render good inhibition efficiency, because the energy to remove an electron from the last occupied orbital will be low [31]. A molecule with a low energy gap is more polarization and is generally associated with the high chemical activity and low kinetic stability and is termed soft molecule [32].

The calculated inhibition efficiencies (Table 2) indicate that ATA and PTA compounds possess the highest inhibition efficiency followed by the PyTA and STSC while PTSC and MMTA show very low inhibition 
efficiency. It is worthy to mention that the calculated inhibition efficiencies agree well with those previously experimentally measured inhibition efficiencies.

The frontier molecule orbital density distributions of the compounds are presented in Figure 1. As seen from the figure, the distributions of HOMO and LUMO were mainly average around the whole compound structure, but the population densities of compounds 'frontier orbitals have a more obvious difference. ATA, PTA PYTA and STSC have higher and more uniform population densities than the MMTA and PTSC. It can be seen that the area of nitrogen and sulfur atoms charged a large electron density and might form adsorption active centers.

Frontier orbital theory is useful in predicting adsorption centers of the inhibitor molecules responsible for the interaction with surface metal atoms [33-34]. Terms involving the frontier MO could provide dominative contribution, because of the inverse dependence of stabilization energy on orbital energy difference [33]. Moreover, the gap between the HOMO and LUMO energy levels of the molecules was another important factor that should be considered. Reportedly, excellent corrosion inhibitors are usually those organic compounds which not only offer electrons to unoccupied orbital of the metal, but also accept free electrons from the metal [33, $35]$.

According to Gece and Bilgiç [36], when the position of $\mathrm{N}$ atoms was changing, the corrosion inhibition efficiency changed. The quantum chemistry calculation revealed that the HOMO energy level of ATAH and PTA was boosted significantly while the energy gap dropped sharply. The linear correlation between MO energy level and the corrosion inhibition efficiency of the tested compounds (Figures 2, 3, and 4) proved that the higher the HOMO energy of the inhibitor, the greater the trend of offering electrons to unoccupied d orbital of the metal and the higher the corrosion inhibition efficiency; in addition, the lower the LUMO energy, the easier the acceptance of electrons from metal surface, as the HOMO-LUMO energy gap decreased and the efficiency of inhibitor improved.

$\mathrm{N}$ atoms were the active center, which had the strongest ability of bonding to the metal surface. On the other side, HOMO was mainly distributed on the areas containing nitrogen and sulfur atoms. Thus, the areas containing $\mathrm{N}$ and $\mathrm{S}$ atoms were probably the primary sites of the bonding. Inhibition efficiency was enhanced by increase in HOMO energy and electron density. It is concluded that the region of active centers transforming electrons from $\mathrm{N}$ atoms to copper surface. The electron configuration of $\mathrm{Cu}$ is [Ar] $3 \mathrm{~d} 104 \mathrm{~s} 1 \mathrm{Ar}$. The $4 \mathrm{~d}$ orbitals are not fully filled with electrons. $\mathrm{N}$ and $\mathrm{O}$ atoms have lonely electron pairs that are important for bonding unfilled $4 \mathrm{~d}$ orbitals of copper atom and determining the adsorption of the molecules on the metal surface. ATAH had the highest inhibition efficiency, which resulted from the geometry change that led to HOMO energy increase and electron density distribution in the molecule.

Based on the discussion above, it can be concluded that the ATAH and PTA molecules have many active centers of negative charge. In addition, the areas containing $\mathrm{N}$ and $\mathrm{S}$ atoms are the most possible sites of bonding metal surface by donating electrons to the metal $\mathrm{Cu}$. Accordingly, we could calculate the quantum chemical parameters before the experiment to forecast the inhibitor effectiveness. This can help improve accuracy and save time.

At present, more and more researchers are engaged in the calculation of the quantum chemistry of the organic heterocyclic inhibitors, especially the nitrogen-heterocyclic inhibitors. El Sayed et al. [37] proceed the quantum chemical calculation for corrosion inhibitions of some triazole, oxadiazole, and thiadiazole derivatives by using a Restricted Hartree-Fock (RHF) method. Arslan et al. [30] took the quantum chemical studies on the corrosion inhibition of efficiency increased with the increase in $E_{\text {номо }}$ and decrease in $E_{\text {номо }}-E_{\text {LUMO }}$. ATAH had the highest inhibition efficiency because it had the highest HOMO energy and it was most capable of offering electrons. The distribution of electronic density shows that the molecules had many negatively charged active centers. The electron density was found to be positively correlated with nitrogen and sulfur atoms, which resulted in increasing inhibition efficiency. The areas containing $\mathrm{N}$ and $\mathrm{S}$ atoms are most possible sites for bonding the metal iron surface by donating electrons to the metal.

Quantum chemical calculations indicated that considering only one parameter is not convenient; hence several parameters or a composite index of more than two or more quantum chemical parameters were taken into consideration to characterize the inhibition activity of the molecules. Quantum chemical calculation approach is adequately sufficient to also forecast the inhibitor effectiveness using the theoretical approach. Moreover, it may be used to find the optimal group of parameters that might predict the structure and molecule suitability to be an inhibitor.

\section{Conclusions}

The relationships between inhibition efficiency of copper and $E_{\mathrm{HOMO}}, \mathrm{E}_{\mathrm{LUMO}}$ and $E_{\mathrm{HOMO}}-E_{\mathrm{LUMO}}$, were calculated by DFT method. It is postulated that the inhibition efficiency is increased with the increase in HOMO, the decrease in LUMO and the increase in the energy gap. Accordingly, the descending order of inhibition efficiency will be as follows: ATA $>$ PTA $>$ PyTA $>$ STSC $>$ PTSC $>$ MMTA. 
The distribution of electronic density shows that the molecules had many negatively charged active centers. The electron density was found to be positively correlated with $\mathrm{N}$ and $\mathrm{S}$ atoms, which resulted in increasing inhibition efficiency. The areas containing $\mathrm{N}$ and $\mathrm{S}$ atoms are most possible sites for bonding the metal iron surface by donating electrons to the metal.

Quantum chemical calculation approach is adequately sufficient to also forecast the inhibitor effectiveness using the theoretical approach. Moreover, it may be used to find the optimal group of parameters that might predict the structure and molecule suitability to be an inhibitor.

\section{References}

[1] C. Kaya et al., "Quantum chemical and molecular dynamics simulation studies on inhibition performances of some thiazole and thiadiazole derivatives against corrosion of iron," vol. 219, pp. 497-504, 2016.

[2] S. N. Raicheva, B. V Aleksiev, and E. I. Sokolova, "The effect of the chemical structure of some nitrogenand sulphur-containing organic compounds on their corrosion inhibiting action," Corros. Sci., vol. 34, no. 2, pp. 343-350, 1993.

[3] S. Kaya, B. Tüzün, C. Kaya, and I. B. Obot, "Determination of corrosion inhibition effects of amino acids: Quantum chemical and molecular dynamic simulation study," J. Taiwan Inst. Chem. Eng., vol. 58, pp. 528-535, 2016.

[4] M. Elayyachy, B. Hammouti, and A. El Idrissi, "New telechelic compounds as corrosion inhibitors for steel in 1M HCl," Appl. Surf. Sci., vol. 249, no. 1, pp. 176-182, 2005.

[5] M. Bouklah, B. Hammouti, M. Lagrenee, and F. Bentiss, "Thermodynamic properties of 2, 5-bis (4methoxyphenyl)-1, 3, 4-oxadiazole as a corrosion inhibitor for mild steel in normal sulfuric acid medium," Corros. Sci., vol. 48, no. 9, pp. 2831-2842, 2006.

[6] M. Ajmal, A. S. Mideen, and M. A. Quraishi, "2-Hydrazino-6-methyl-benzothiazole as an effective inhibitor for the corrosion of mild steel in acidic solutions," Corros. Sci., vol. 36, no. 1, pp. 79-84, 1994.

[7] N. Caner and I. A. N. Love, "Quantum Chemical Studies of Some Rhodanine Azosulpha Drugs as Corrosion Inhibitors for Mild Steel in,” vol. 110, no. 2010, pp. 1003-1018, 2009.

[8] F. Bentiss, M. Lebrini, and M. Lagrenée, "Thermodynamic characterization of metal dissolution and inhibitor adsorption processes in mild steel/2, 5-bis (n-thienyl)-1, 3, 4-thiadiazoles/hydrochloric acid system," Corros. Sci., vol. 47, no. 12, pp. 2915-2931, 2005.

[9] D. Wang, S. Li, Y. Ying, M. Wang, H. Xiao, and Z. Chen, "Theoretical and experimental studies of structure and inhibition efficiency of imidazoline derivatives," Corros. Sci., vol. 41, no. 10, pp. 19111919, 1999.

[10] I. A. N. Love, C. E. M. Il, and A. Umoren, "Theoretical Studies of Some Sulphonamides as Corrosion Inhibitors for Mild Steel in Acidic Medium,” vol. 110, pp. 2614-2636, 2010.

[11] M. Lebrini, M. Traisnel, M. Lagrenée, B. Mernari, and F. Bentiss, "Inhibitive properties, adsorption and a theoretical study of 3,5- bis(n-pyridyl)-4-amino-1,2,4-triazoles as corrosion inhibitors for mild steel in perchloric acid," Corrosion Science, vol. 50, no.2, pp. 473-479, 2008.

[12] H. Ju, Z.-P. Kai, and Y. Li, "Aminic nitrogen-bearing polydentate Schiff base compounds as corrosion inhibitors for iron in acidic media: a quantum chemical calculation," Corrosion Science, vol. 50, no. 3, pp. 865-871, 2008.

[13] B. Liu, H. Xi, Z. Li, and Q. Xia, "Adsorption and corrosion- inhibiting effect of 2-(2-[2-(4Pyridylcarbonyl) hydrazono] methylphenoxy)acetic acid on mild steel surface in seawater," Applied Surface Science, vol. 258, no. 17, pp. 6679-6687, 2012.

[14] K. Bhrara and G. Singh, "Adsorption kinetics of benzyl triphenyl phosphonium chloride on mild steel in 1N sulphuric acid," Surface Engineering, vol. 21, no. 3, pp. 165-171, 2005.

[15] E.Szocs, G.Vastag, A.Shaban and E.Kalman, Corros. Sci., 47 (2005) 893.

[16] B.V. Appa Rao, K. Chaitanya Kumar, Arabian Journal of Chemistry, 10 (2) (2017) s2245.

[17] N. Kovačević, I. Milošev, A. Kokalj, Corros. Sci., 124 (2017) 25.

[18] E. S. H. El Ashry, A. El Nemr, S. A. Esawy, and S. Ragab, "Corrosion inhibitors Part II: Quantum chemical studies on the corrosion inhibitions of steel in acidic medium by some triazole, oxadiazole and thiadiazole derivatives," Electrochim. Acta, vol. 51, no. 19, pp. 3957-3968, 2006.

[19] J. Fang and J. Li, "Quantum chemistry study on the relationship between molecular structure and corrosion inhibition efficiency of amides,” J. Mol. Struct. THEOCHEM, vol. 593, no. 1, pp. 179-185, 2002.

[20] F. Kandemirli, Seda Sağdınç"Theoretical study of corrosion inhibition of amides and thiosemicarbazones," vol. 49, pp. 2118-2130, 2007.

[21] A. Yurt, G. Bereket, and C. Ogretir, "Quantum chemical studies on inhibition effect of amino acids and hydroxy carboxylic acids on pitting corrosion of aluminium alloy 7075 in NaCl solution,” J. Mol. Struct. THEOCHEM, vol. 725, no. 1, pp. 215-221, 2005. 
[22] H. Ma, S. Chen, Z. Liu, and Y. Sun, "Theoretical elucidation on the inhibition mechanism of pyridine-pyrazole compound: A Hartree Fock study,” J. Mol. Struct. THEOCHEM, vol. 774, no. 1, pp. 19-22, 2006.

[23] T. Arslan, F. Kandemirli, E. E. Ebenso, I. Love, and H. Alemu, "Quantum chemical studies on the corrosion inhibition of some sulphonamides on mild steel in acidic medium," Corros. Sci., vol. 51, no. 1, pp. 35-47, 2009.

[24] M. A. Amin et al., "Monitoring corrosion and corrosion control of iron in $\mathrm{HCl}$ by non-ionic surfactants of the TRITON-X series - Part III . Immersion time effects and theoretical studies," Corros. Sci., vol. 53, no. 5, pp. 1895-1909, 2011.

[25] M. Lebrini, F. Robert, H. Vezin, and C. Roos, "Electrochemical and quantum chemical studies of some indole derivatives as corrosion inhibitors for C38 steel in molar hydrochloric acid," Corros. Sci., vol. 52, no. 10 , pp. 3367-3376, 2010.

[26] I. B. Obot and N. O. Obi-Egbedi, "Theoretical study of benzimidazole and its derivatives and their potential activity as corrosion inhibitors," Corros. Sci., vol. 52, no. 2, pp. 657-660, 2010.

[27] D. K. Yadav, B. Maiti, and M. A. Quraishi, "Electrochemical and quantum chemical studies of 3, 4dihydropyrimidin-2 $(1 \mathrm{H})$-ones as corrosion inhibitors for mild steel in hydrochloric acid solution," Corros. Sci., vol. 52, no. 11, pp. 3586-3598, 2010.

[28] D. Özkır, K. Kayakırılmaz, E. Bayol, A. A. Gürten, and F. Kandemirli, “The inhibition effect of Azure A on mild steel in $1 \mathrm{M} \mathrm{HCl}$. A complete study: Adsorption, temperature, duration and quantum chemical aspects," vol. 56, pp. 143-152, 2012.

[29] E. E. Ebenso, M. M. Kabanda, T. Arslan, M. Saracoglu, and F. Kandemirli, "Quantum Chemical Investigations on Quinoline Derivatives as Effective Corrosion Inhibitors for Mild Steel in Acidic Medium," vol. 7, pp. 5643-5676, 2012.

[30] T. Arslan, F. Kandemirli, E.E. Ebenso, I. Love, H. Alemu, "Quantumchemical studies on the corrosion inhibition of some sulphonamides on mild steel in acidic medium," Corrosion Science,vol.51,no.1,pp.3547,2009.

[31] D. K. Yadav, B. Maiti, and M. A. Quraishi, "Electrochemical and quantum chemical studies of 3, 4dihydropyrimidin-2 $(1 \mathrm{H})$-ones as corrosion inhibitors for mild steel in hydrochloric acid solution," Corros. Sci., vol. 52, no. 11, pp. 3586-3598, 2010.

[32] M. Bouklah, B. Hammouti, M. Lagrenee, and F. Bentiss, "Thermodynamic properties of 2, 5-bis (4methoxyphenyl)-1, 3, 4-oxadiazole as a corrosion inhibitor for mild steel in normal sulfuric acid medium," Corros. Sci., vol. 48, no. 9, pp. 2831-2842, 2006.

[33] E.Szocs, G.Vastag, A.Shaban and E.Kalman, Electrochemical behaviour of an inhibitor film formed on copper surface, Corros. Sci., 47 (2005) 893.

[34] M. Lebrini, M. Traisnel, M. Lagrenée, B. Mernari, and F. Bentiss, "Inhibitive properties, adsorption and a theoretical study of 3,5- bis(n-pyridyl)-4-amino-1,2,4-triazoles as corrosion inhibitors for mild steel in perchloric acid," Corrosion Science, vol. 50, no.2, pp. 473-479, 2008.

[35] M.Lashgari,M.R.Arshadi,andG.A.Parsafar,"Asimpleand fast method for comparison of corrosion inhibition power between pairs of pyridine derivative molecules," Corrosion, vol. 61, no. 8, pp. 778-783, 2005

[36] G. Gece and S. Bilgic, "A theoretical study on the inhibition efficiencies of some amino acids as corrosion inhibitors of nickel," Corrosion Science,vol.52,no.10,pp.3435-3443,2010.

[37] ElSayed, H.ElAshry,A.EINemr,S.A.Esawy,andS.Ragab, "Corrosion inhibitors: part II: quantum chemical studies on the corrosion inhibitions of steel in acidic medium by some triazole, oxadiazole and thiadiazole derivatives," Electrochimica Acta,vol.51,no.19,pp.3957-3968,2006. 
Table 1: The calculated parameters of the tested inhibitors by B3LYP/6-311++G(2d,2p)

\begin{tabular}{|c|c|c|c|c|c|c|c|c|c|c|}
\hline Inhibitor & Номо & LUMO & $\begin{array}{c}\text { Energy } \\
\text { gap }\end{array}$ & Hardness & Softness & $\begin{array}{c}\text { Electronegati } \\
\text { vity }\end{array}$ & $\begin{array}{l}\text { Chemical } \\
\text { potential }\end{array}$ & $\begin{array}{l}\text { Electrophilicity } \\
\text { index }(\omega)\end{array}$ & $\begin{array}{l}(\mu+\eta)(\mu \\
+\eta) / 2 \eta\end{array}$ & $\begin{array}{c}(\mu-\eta)(\mu-\eta \\
) / 2 \eta\end{array}$ \\
\hline PyTA & -7.633 & -2.233 & 5.400 & 2.700 & 0.185 & 4.933 & -4.933 & 4.507 & 0.924 & 10.790 \\
\hline PyTA. $\mathrm{H}_{2} \mathrm{O}$ & -7.396 & -1.926 & 5.470 & 2.735 & 0.183 & 4.661 & -4.661 & 3.972 & 0.678 & 10.001 \\
\hline ATA & -7.345 & -1.003 & 6.342 & 3.171 & 0.158 & 4.174 & -4.174 & 2.747 & 0.159 & 8.506 \\
\hline MMTA & -6.357 & -2.492 & 3.865 & 1.933 & 0.259 & 4.424 & -4.424 & 5.064 & 1.606 & 10.455 \\
\hline MTA. $\mathrm{H}_{2} \mathrm{O}$ & -6.360 & -2.091 & 4.269 & 2.135 & 0.234 & 4.225 & -4.225 & 4.182 & 1.024 & 9.474 \\
\hline PTSC & -5.995 & -1.052 & 4.942 & 2.471 & 0.202 & 3.523 & -3.523 & 2.512 & 0.224 & 7.271 \\
\hline PTSC. $\mathrm{H}_{2} \mathrm{O}$ & -6.165 & -1.074 & 5.091 & 2.604 & 0.192 & 3.561 & -3.561 & 2.435 & 0.176 & 7.298 \\
\hline PTAH. $\mathrm{H}_{2} \mathrm{O}$ & -6.913 & -1.430 & 5.484 & 2.742 & 0.182 & 4.172 & -4.172 & 3.173 & 0.373 & 8.716 \\
\hline STSC & -6.062 & -1.099 & 4.963 & 1.720 & 0.291 & 4.342 & -4.342 & 5.481 & 1.999 & 10.683 \\
\hline STSC. $\mathrm{H}_{2} \mathrm{O}$ & -6.173 & -1.076 & 5.097 & 1.725 & 0.290 & 4.210 & -4.210 & 5.136 & 1.789 & 10.208 \\
\hline
\end{tabular}

Table 2: Calculated inhibition efficiency of the tested inhibitors

\begin{tabular}{|c|c|c|}
\hline rb3lyp/6-311++g(2d,2p) & $\beta$-Total & IE\% \\
\hline РуТАН & 4.509812318 & 88.79 \\
\hline ATAH & 1.044035608 & 91.95 \\
\hline MMTAH & 6.66192446 & 61.79 \\
\hline PTSC & 31.46131605 & 16.23 \\
\hline PTA & 0.380956092 & 93.39 \\
\hline STSC & 62.04153687 & 81.91 \\
\hline PyTAH. $\mathrm{H}_{2} \mathrm{O}$ & 4.563211865 & 88.79 \\
\hline ATAH. $\mathrm{H}_{2} \mathrm{O}$ & 1.918086544 & 91.95 \\
\hline MTAH. $\mathrm{H}_{2} \mathrm{O}$ & 27.16808403 & 61.79 \\
\hline PTSC- $\mathrm{H}_{2} \mathrm{O}$ & 3.152882926 & 16.25 \\
\hline PTA- $\mathrm{H}_{2} \mathrm{O}$ & 0.405493675 & 93.45 \\
\hline STSC- $\mathrm{H}_{2} \mathrm{O}$ & 21.15160409 & 81.96 \\
\hline
\end{tabular}




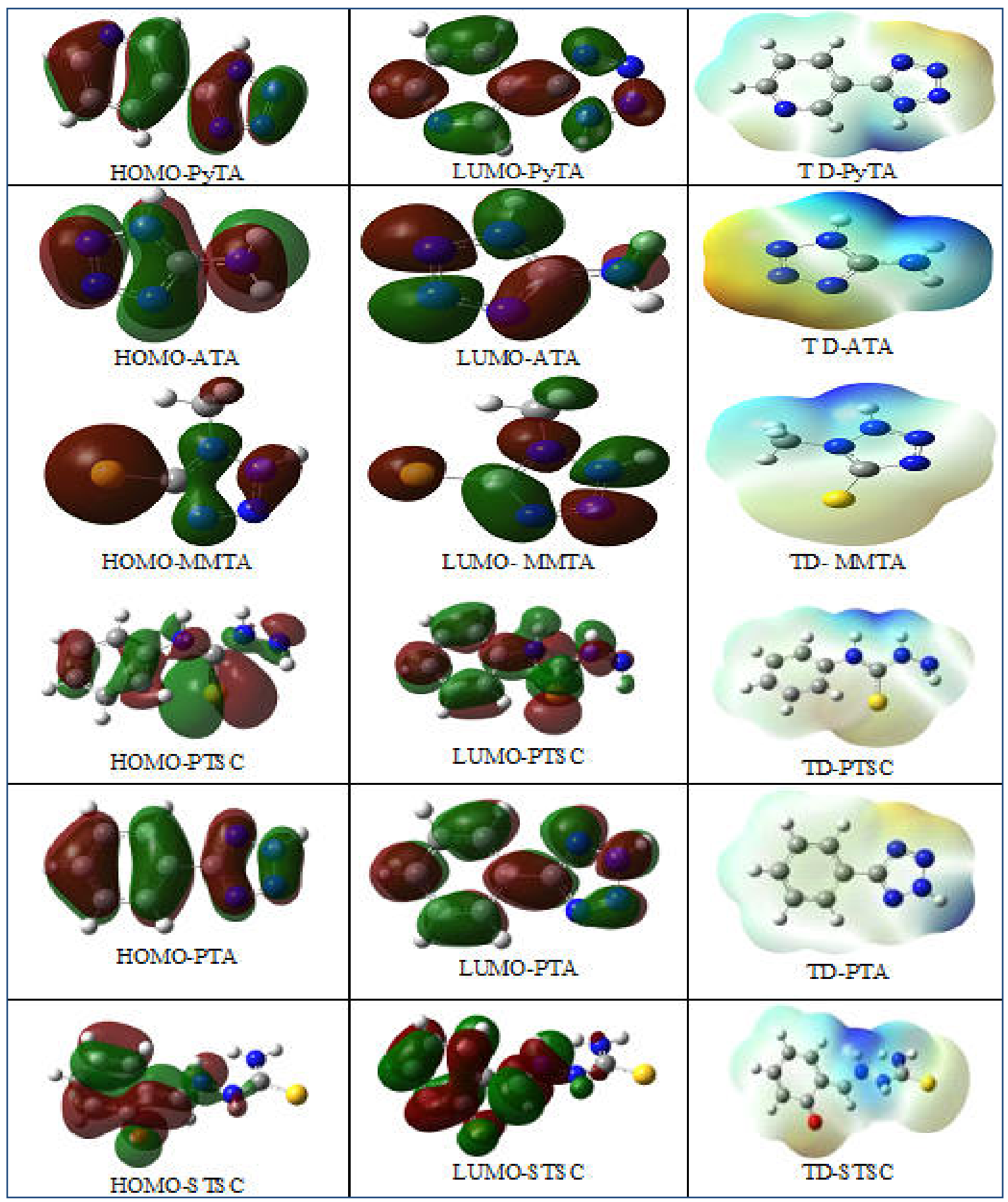

Fig.1: Molecular structure, HOMO, LUMO, OPT \&TOTAL DENSITY of PyTA, ATA, MMTA, PTSC, PTA and STSC molecules 


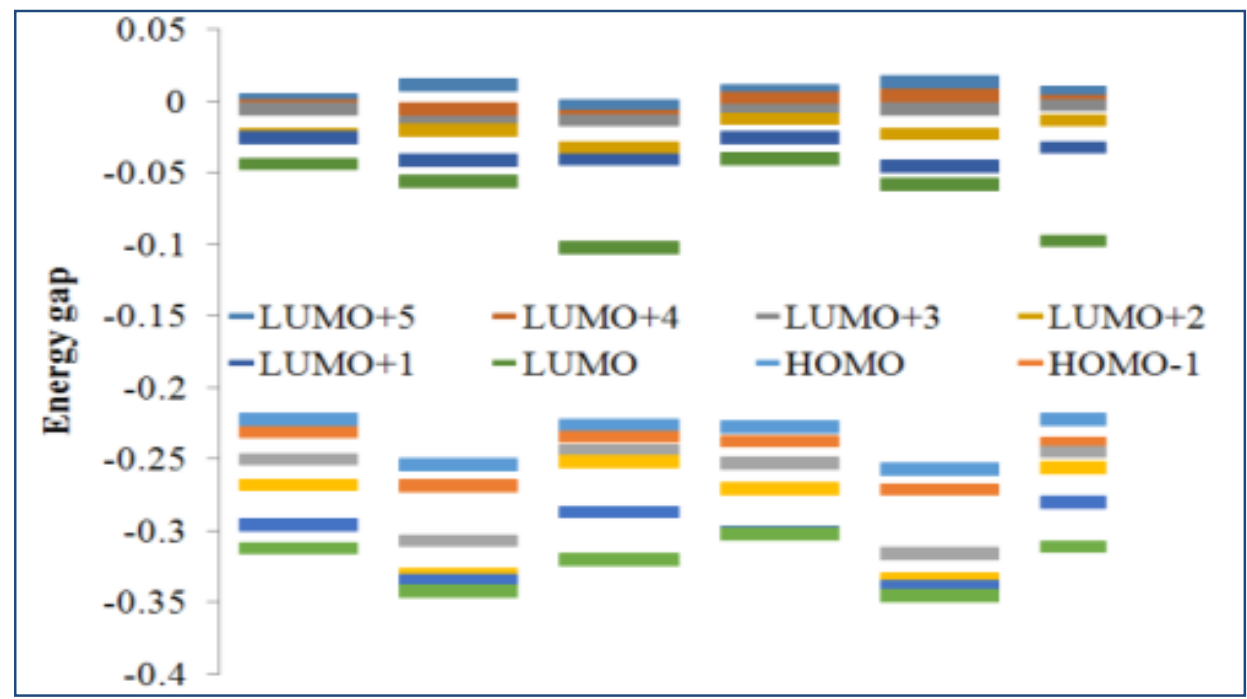

Figure 2: HOMO \& LUMO of PTSC, PTA and STSC inhibitors at B3LYP/6-311++G (2d, 2p)

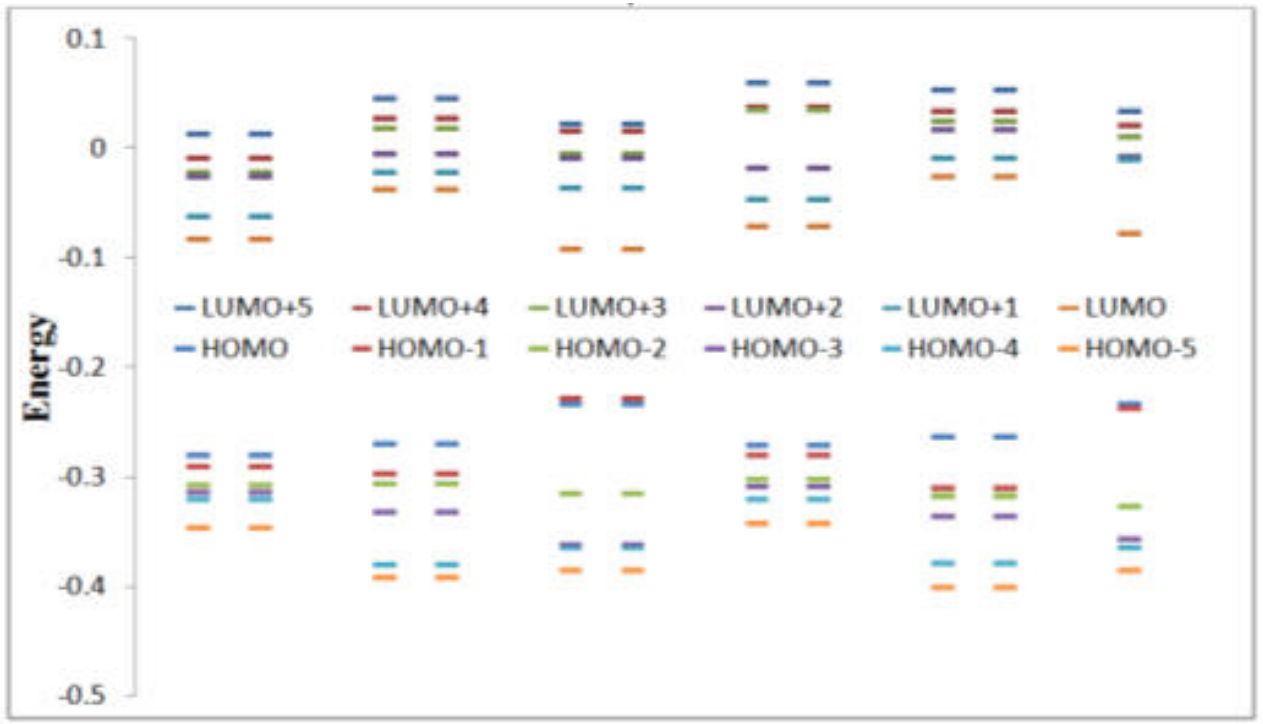

Figure 3: HOMO \& LUMO of PyTA, ATA and MMTA, at B3LYP/6-311++G (2d, 2p) 